I.M. AMETOV' and S.V. KALUZHNY|

1 VNlineft, c/o 10 Dmitrovsky Proezd, 125422 Moscow, Russia

${ }^{2}$ Moscow State University

\title{
Introduction
}

At present time MEOR is considered as one of the most promising methods. But though research in this area have been already conducting for several decades, these methods have not reached large scale application in spite of many positive laboratory and especially field tests results. However: their obvious advantages such as: ecological safety: reasonable expenses, high effectiveness shown by results of laboratory and severa! field tests, support the attraction of MEOR. At the first place it is necessary to estimate whether these methods stimulate the oil displacement as a whole or only the bottom hole zone or they combine both. What are the determining reasons of the effectiveness (or ineffectiveness) of MEOR implementation? The analysiș. of well known works in this area shows that main attention was directed to determine the conditions for optimization of microbial activity and hence, uptake of metabolic products with taking into account of natural conditions and external technological factors. Taking this into consideration there were estimated in natural and laboratory conditions such characteristics as displacement efficiency, additional oil production, sweep efficiency etc. At the same time technological aspects of the proper problem and specifically the peculiarities of hydrodynamic processes, such as the porous medium structure, surface properties of the reservoir are practically ignored. Taking this into account the purpose of the report is a quantitative evaluation of possible influence of microbial vital activity on physical-chemical phenomena in processes of oil displacement from porous medium, as well as on discrimination of possible mechanisms of microorganisms influence on filtration process and discussion of interpretation peculiarities of laboratory and natural experiments results.

\section{Numerical estimation}

The MEOR application is based on conclusion that availability of microorganisms and their metabolic products improve conditions of oil displacement from porous medium. As main factors it is considered: production of surfactants, gases, acids, mechanical reduction of porous medium permeability by microorganisms. In this case it is supposed that the mechanism of microbial stimulation of ofl recovery process is analogous to well known EOR 
methods such as application of surfactant, alkali and acid solutions, water gas stimulation and so on. However simple estimations show that this analogy has only quaitative character and could not explain the observed effect of microbial stimulation. The results of quantitative comparison of each of these factors with analogous known EOR methods are presented below (tabl 1). It is also necessary to discuss the question about relative dimensions of hacteria and pore channels. Bacterial dimensions have the value approximately $10^{-6}-10^{-7} \mathrm{~m}$, that is comparable with average pore dimensions of majority reservoirs. It means that

bacterial transport along the porous medium in the conditions of real resenoir will be difficult. In the consequence of that, when permeability declines, this effect increases and, in case of great value of permeability EOR, problems aren't so actual, as for low permeability reservoirs. Therefor the effectiveness of MEOR application is strongly determined by reservoir characteristics.

\section{Experiments}

Special experiments for qualitative estimation of influence of porous medium parameters on effectiveness of microbial technology have been carried out. By this, permeabilities taken for two experiment series were selected essentially different, but with average pore size less than bacterial dimensions. Each series included experiments on three models of porous media with similar value of permeability, one of those was a control. The permeability of samples of the first serie was approximately $200 \mathrm{md}$, in the second was 390 md. Each experiment included oil displacement by water up to achievement residual oil saturation, injection of 0.5 pore volume of nutrient composition 3 days shut-up of model and followed by displacement. Last cycle was repeated several times. In control experiments, the displacement in both cases was carried out by Raimond medium. For comparison, the second model of the first serie was injected $n .1$ pore volume of nutritions composition. Results are presented in table 2.

Table :

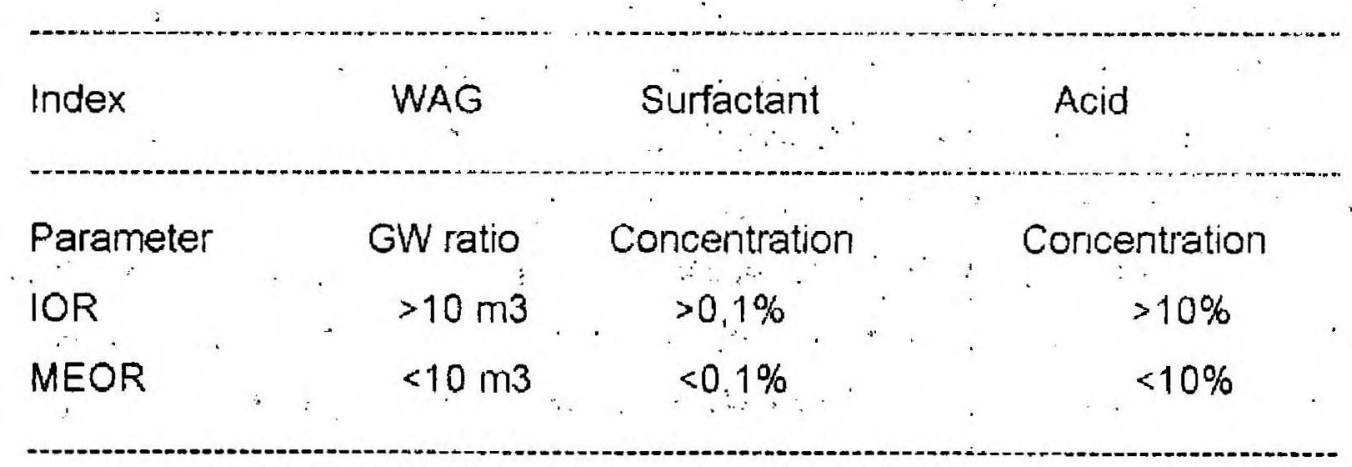




\begin{tabular}{|c|c|c|c|c|c|c|}
\hline Displacement & & 1 series & & & 2 series & \\
\hline efficiency & 1 samp & 2 samp & 3 samp & 1 samp & 2 samp & 3 samp \\
\hline $\begin{array}{l}\text { Water displa- } \\
\text { cement }\end{array}$ & 0.650 & 0.683 & 0.656 & 0.574 & 0.514 & 0.600 \\
\hline 1 stage & $\begin{array}{c}0.664 \\
\vdots\end{array}$ & 0.693 & & 0.687 & 0.639 & \\
\hline 3 stage & 0.676 & 0.701 & 0.660 & 0.713 & 0.668 & 0.603 \\
\hline
\end{tabular}

The results of the table show that process effectiveness is essentially higher in the second series than in the first one, where permeability is lower. This is likely accounted for that in the medium with great permeability pore channel sizes are larger and therefore there are more opportunities for microorganism travel that results to better sweep by stimulation.

Experiments analysis: It was not taken into account in the examples above described that the real porous media are not monodisperie, but they were characterized by the function of pore size distribution. Three experimentally determined furictions of distribution for porous media of different lithology composition are presented on the fig 1 . In spite of the fact that all of them have approximately similar permeability, it is seen, that the first case is the most favourable for microorganism travel. This fact usually has not been taken into account in MEOR research.

The porous medium parameters could also influence the microbial stimulation process by another way. It is known that bacteria have, in general, hydrophobic surfaces. That's why they can change surface rock properties and make the rock more hydrophobic the porous medium. At these conditions there will take place oil phase redistribution in direction to small pore channels and the displacement will be difficult. This process seems to be more perceptible in low-permeability reservoirs. Simple calculation shows that equivalent specific surface of bacterial colony is comparable with specific porous medium surface.

Thus, it is possible to distinguish three main mechanisms of stimulation the oil displacement by microorganisms: 1 - pore channels clogging by microorganisms, that leads to redistribution regions in porous medium and to inflow or injection profile modification; 2 - metobolic products stimulation on oil and rock; 3 making hydrophobization of the surface of reservoir. Based on the analysis described above, one can conclude the MEOR is hardly occurred oil displacement process it is difficult to do it in the volume of whole reservoir. It is explained by both the difficulties of bacterial transport into the formation depth and low concentration of metobolic products formed. Above 
mentioned estimations were conducted for conditions of the whole reservoir. However, when recalculating for limited volume of bottom hole zone conditions stimulation effectiveness sharply increases.

\section{Discussion}

Bottom hole zone can be considered as a localized zone of bacterial activity development, to where substances necessary for bacterial activity are supplied from the surface. The metobolic products are transferred by filtration flow into formation depth and their concentration sharply declines with increase of the distance from well as for account of sweeping stimulation volume increasing as because of the ratio between "technology" time and "microbiology " time is increasing. This fact is usually not taken into account in interpretation of field tests results. The doubling time of bacteria usually varies from 1 to 10 days. For this period microorganisms are transferred by filtration flow by few meters.distance, that is sufficiently less than the distance between injection and production wells.

According to conducted analysis, one can distinguish two stages in the microbial stimulation process. At the first stage there is stimulation of the bottom hole zones and injection profiles modified. This leads to increase the effectiveness of flooding process and to increase of oil recovery. On the second stage, bacteria migrate into the formation depth. This stage, of the process sufficiently depends on rock lithology properties and it obviously has less influence on whole process. If not taking into account microbial process properties, stimulation effectiveness, as above mentioned, sufficiently depends on formation depth, pore space

structure and hydrodynamic regimes of stimulation.

\section{Field testing}

Above mentioned considerations are confirmed by biotechnology pilot tests at Vayngapurovskoye field conducted for two years. Injection volume of nutrient medium was $270 \mathrm{~m}^{3}$. Injection was carried out through one injection well. Well injectivity was about $200 \mathrm{~m}^{3} /$ day. Volume of formation element, where the experiment was carried out, can be estimated as $1.2 * 10^{6} \mathrm{~m}^{3}$. During the experiment, the composition of formation and injected water was monitored by taking samples every $7-10$ days during composition injection and two times per month after its stopping. In addition back flow of injection well took place.Producing wells began to react on the biocomposition injection in five months after its start. Maximum effect was received in 8-9 months. Analysis of the pilot tests results allows to make following conclusions. As five months passed from nutrient medium injection to the start of reaction, the formation volume swept by stimulation was not more than $2-3 \%$ from pilot area volume. It means that achieved average reduce of $3 \%$ of production wells water cutting, production of produced water cut down by $34000 \mathrm{~m}^{3}$ and oil production increase by $2700 \mathrm{~m}^{3}$ occurred. Due to the stimulation took place in the bottom hole zone of injection well N726. Some results are presented on the Fig 2. 
Above mentioned analysis allows to formulate the main conception about the process of microbial stimulation. It is the principle moment because it gives the possibility of more effective selection, planning and optimization of MEOR. Microbial stimulation can be made both in injection and in production wells. By that, microorganism types should be selected both by formation conditions, particularly in bottom hole zone, and specific aims of stimulation.

In conclusion, it should be noted that the possibilities of MEOR can be significantly tended by combination with known EOR. At present time, such combined technologies began to develop in VNIIneft for example, technologies of microbial, watergas and micronuclear stimulation on reservoirs. Preliminary results seem to be promising. 


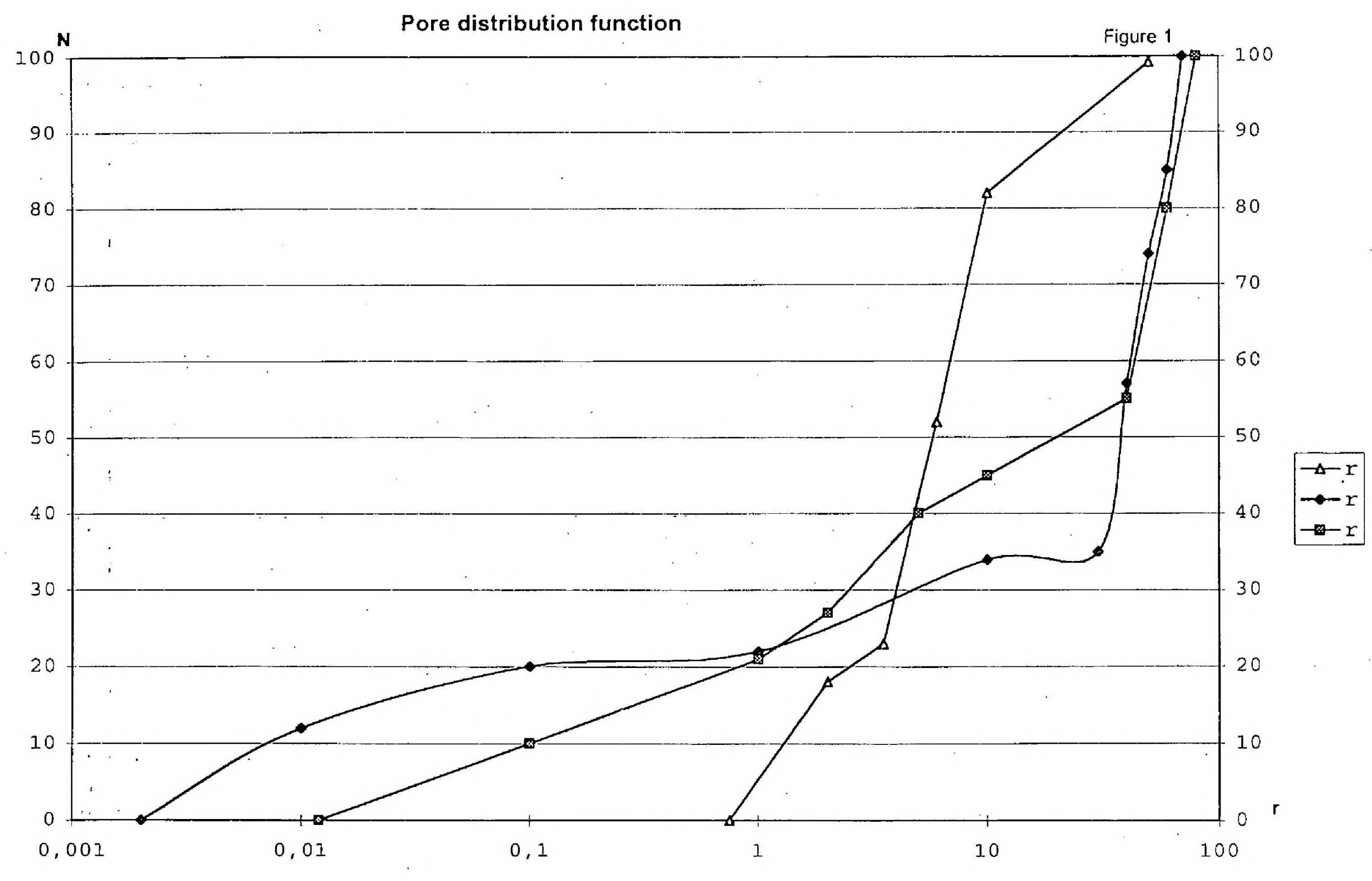




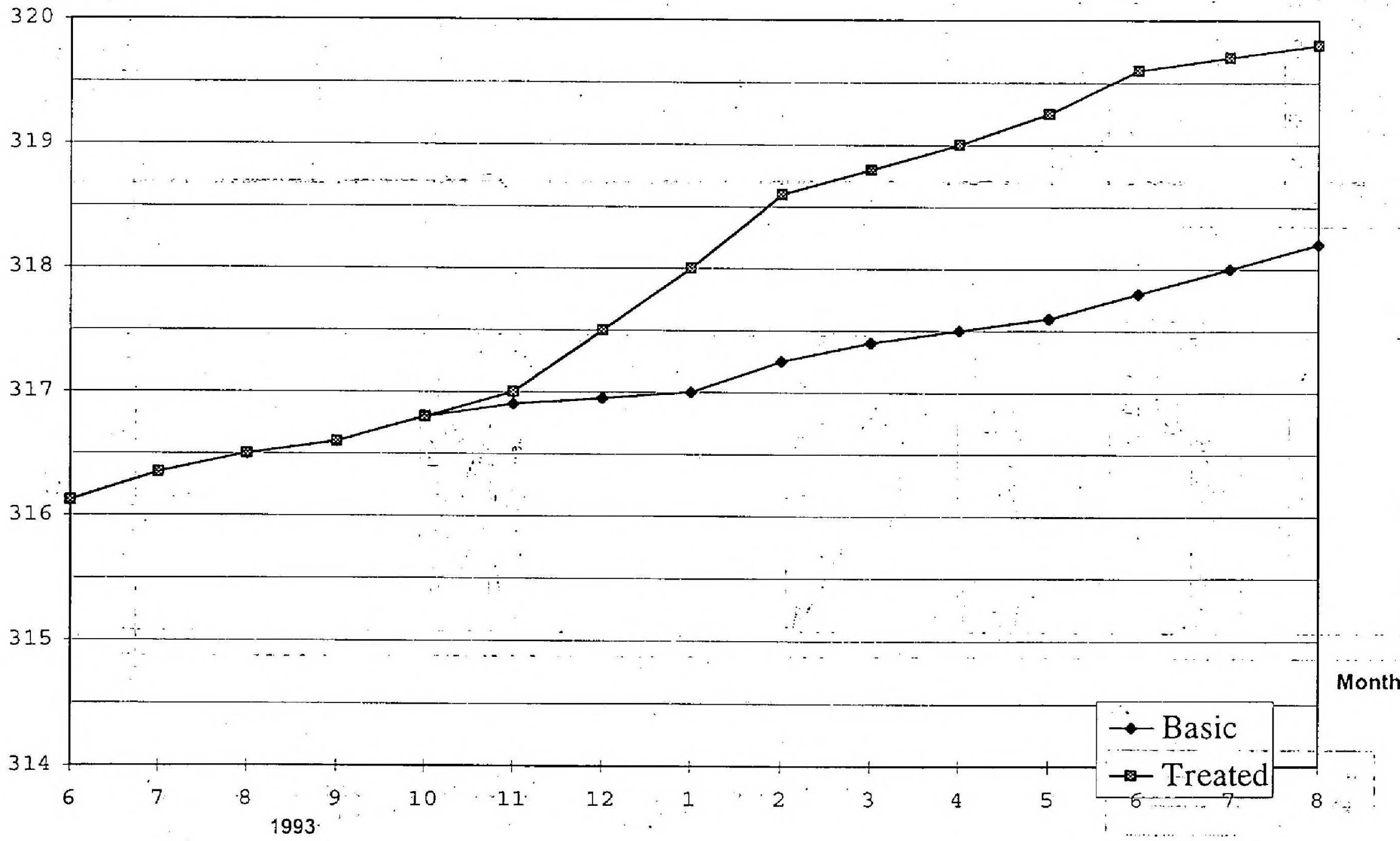

